\title{
Aberrant expression of sperm-specific glycolytic enzymes are associated with poor sperm quality
}

\author{
XUEXIA LIU ${ }^{*}$, QIAN LI ${ }^{2 *}$, WENJUAN WANG ${ }^{3}$ and FUJUN LIU ${ }^{1}$ \\ ${ }^{1}$ Central Laboratory, ${ }^{2}$ Research Department and ${ }^{3}$ Reproduction Medical Center, \\ The Affiliated Yantai Yuhuangding Hospital of Qingdao University, Yantai, Shandong 264000, P.R. China
}

Received June 19, 2018; Accepted December 18, 2018

DOI: $10.3892 / \mathrm{mmr} .2019 .9926$

\begin{abstract}
The energy required for normal sperm function is mainly generated by glycolysis and oxidative phosphorylation. Testis-specific glycolytic enzymes are expressed in germ cells and present in mature spermatozoa. The objective of the present study was to investigate the association between aberrant expression of glycolytic enzymes and human sperm quality. In silico analysis of glyceraldehyde-3-phosphate dehydrogenase, testis-specific (GAPDHS), phosphoglycerate kinase 2 (PGK2) and lactate dehydrogenase (LDHC) identified that they were exclusively expressed in post-meiotic germ cells and this was validated in human testes using immunohistochemistry. Compared with the testes of young adults, markedly lower expression levels of these glycolytic enzymes were observed in the testes of elderly adults. Similarly, low levels were observed in immature and asthenozoospermic spermatozoa. The expression levels of GAPDHS, PGK2 and LDHC in the spermatozoa were closely correlated with progressive sperm motility. The results indicated that the expression of GAPDHS, PGK2 and LDHC in sperm may be associated with sperm quality, and there may be a similar molecular mechanism underlying sperm quality in immature and asthenozoospermic spermatozoa. This study reveals a close association of glycolytic enzymes with sperm quality. The data may greatly contribute to the molecular evaluation of sperm quality and the diagnosis and treatment of asthenozoospermia.
\end{abstract}

\section{Introduction}

Infertility is a worldwide reproductive health problem that affects $\sim 15 \%$ of couples, with the male responsible for approximately half of cases $(1,2)$. Asthenozoospermia, a common

Correspondence to: Dr Fujun Liu, Central Laboratory, The Affiliated Yantai Yuhuangding Hospital of Qingdao University, 20 Yuhuangding East Road, Yantai, Shandong 264000, P.R. China E-mail: 1fjyt@126.com

${ }^{*}$ Contributed equally

Key words: testis, sperm quality, spermatozoa, motility, energy cause of male infertility, is characterized by reduced sperm motility in fresh ejaculate, which prevents the sperm from moving towards and penetrating the egg and results in infertility $(3,4)$. In mammals, the generation of spermatozoa that are capable of fertilization is a highly complex process that includes spermatogenesis in the testis and sperm maturation in the epididymis (5). During these processes, sperm require adenosine triphosphate (ATP) that is produced by mitochondrial oxidative phosphorylation in the middle piece of the sperm flagellum and by glycolysis in the principal piece of the sperm flagellum in order to provide energy for motility. Glycolysis is essential for sperm motility and male fertility, rather than mitochondrial metabolism (6). A total of three testis-specific glycolytic enzymes, glyceraldehyde-3-phosphate dehydrogenase, testis-specific (GAPDHS), phosphoglycerate kinase 2 (PGK2) and lactate dehydrogenase C (LDHC), known to be involved in glycolysis in spermatozoa (7-9). GAPDHS acts between the ATP-consuming and ATP-generating segments of glycolysis. PGK2 catalyzes the first ATP-generating step in the central metabolic pathway of glycolysis. Conversion of pyruvate to lactate with the concomitant oxidation of nicotinamide adenine dinucleotide (NADH) to $\mathrm{NAD}^{+}$is essential for the continued production of ATP by glycolysis and this reaction is catalyzed by LDH. LDHC is a unique isozyme of LDH that is specifically expressed in germ cells $(10,11)$ and is abundant in spermatids and spermatozoa (12). In studies using knockout models, these three enzymes have been identified to be associated with sperm motility and male fertility (13-15). In the authors' previous study, it wasidentified that PGK2 is closely associated with sperm quality (9); PGK2 was downregulated in spermatozoa from elderly adults and asthenozoospermic patients, suggesting that there is a common mechanism underlying poor sperm quality. However, this molecular mechanism and the associationwith glycolysis remains unclear.

Normal semen specimens contain a certain proportion of abnormal sperm with defective motility and morphology. A high percentage of abnormal sperm is diagnosed as asthenozoospermia. It was hypothesized that fertile males also produce poor-quality sperm (immature sperm) and the underlying mechanism of the poor sperm quality maybe similar to that in infertile men. Therefore, the differential expression of GAPDHS, PGK2 and LDHC were analyzed in the testes of young and elderly males in the present study, and their expression levels in normal spermatozoa, immature spermatozoa and 
asthenozoospermic spermatozoa were evaluated. The results revealed similar reduced expression patterns of GAPDHS, PGK2 and LDHC in the poor-quality sperm, which suggested that there may be a common mechanism responsible for sperm quality that involves glycolysis. The present study provided novel insights for understanding sperm motility regulation and the causes of male infertility.

\section{Materials and methods}

Ethics statement. This study was approved by the ethics committee of Yantai Yuhuangding Hospital (Yantai, China). Written informed consent was signed by participants or their relatives.

In silico analysis. Gene expression profiles of the GDS3113 dataset were extracted from the Gene expression Omnibus (GEO) database (https://www.ncbi.nlm.nih. gov/sites/GDSbrowser?acc=GDS3113), which included the gene expression values of different human tissues. The expression values of GAPDHS, PGK 2 and LDHC in the main tissues (heart, kidney, liver, lung, prostate, spleen and testis) were downloaded and re-compared. The GDS2390 (https://www. ncbi.nlm.nih.gov/sites/GDSbrowser?acc=GDS2390) dataset included the gene expression values of different male mouse germ cells (type A and type B spermatogonia, spermatocytes and round spermatids).

Sample preparation. Human testicular specimens were collected from Yantai Yuhuangding Hospital between March 2016 and December 2017. Human testes were obtained from five young adults (age, 27-36 years) who had died in car accidents. None of them exhibited a history of pathologies that could affect reproductive function and all had previously indicated a willingness to donate their bodies to medical research. The donation of their organs for medical research was approved by their immediate family members. Aged testes samples were obtained from five elderly adults (age, 78-82 years) with prostate cancer who had undergone testicular castration. None of them had undergone anti-androgen treatment and all had normal or mixed spermatogenesis (Johnsen score $>6$ ) and provided written informed consent. Testis score evaluation was performed according to Bergmann and Kliesch (16). All procedures were approved by the Ethics Committee of Yantai Yuhuangding Hospital.

Human semen specimens were collected from Yantai Yuhuangding Hospital between January 2017 and December 2017. Semen samples were collected from 15 healthy young adults males (age, 28-36 years) and 15 patients with asthenozoospermia (age, 25-36 years; progressive motility, $<32 \%$ ). Semen was obtained by masturbation following 7 days of sexual abstinence. Collection and processing of semen samples were conducted in accordance with the World Health Organization's (WHO) Laboratory Manual for the Examination and Processing of Human Semen (5th edition, 2010). Spermatozoa were used for quantitative immunofluorescence experiments and western blot analysis. All sperm donors gave written informed consent when donating the sperm ejaculates for the purposes of the research project. All procedures were approved by the Ethics Committee of Yantai Yuhuangding Hospital.
For preparing the corresponding 80\% Percoll sperm fraction (17), an aliquot of the same semen sample was centrifuged through $2.0 \mathrm{ml}$ of an $80 \%$ single-phase Percoll gradient at $500 \mathrm{x} \mathrm{g}$ for $20 \mathrm{~min}$ at room temperature to obtain mature sperm. The sperm pellet was resuspended in $2 \mathrm{ml}$ PBS and re-centrifuged at $600 \mathrm{x} \mathrm{g}$ for $10 \mathrm{~min}$ at room temperature to eliminate the residual Percoll. The rest of the semen sample was used to obtain immature sperm. After diluting in PBS and centrifuging at $800 \mathrm{x}$ g for $10 \mathrm{~min}$ at room temperature, the supernatant was discarded and the sperm pellet was re-suspended in PBS by gentle pipetting prior to centrifugation at $500 \mathrm{x} \mathrm{g}$ for $10 \mathrm{~min}$ at room temperature and washed with PBS three times.

Immunohistochemistry. Immunohistochemistry was performed to detect the cellular expression of GAPDHS, PGK2 or LDHC in the human testes. As described previously (18), the testicular tissues were fixed with Bouin's solution at room temperature for $12 \mathrm{~h}$ and then embedded in paraffin according to conventional methods. Subsequently, $4-\mu \mathrm{m}$-thick paraffin-sections were prepared. Sections were dewaxed in xylene, rehydrated in descending ethanol series (100, 95 and $80 \%$ ) and washed with ultrapure water. Antigen retrieval was performed for $20 \mathrm{~min}$ in microwave (above $94^{\circ} \mathrm{C}$ ). Following three washes with phosphate buffer saline (PBS), endogenous peroxidases were inhibited by incubation with $3 \%(\mathrm{v} / \mathrm{v})$ $\mathrm{H}_{2} \mathrm{O}_{2}$ for $10 \mathrm{~min}$. The sections were washed with PBS three times and blocked in $3 \%(\mathrm{v} / \mathrm{v})$ bovine serum albumin (BSA; Sigma-Aldrich; Merck KGaA, Darmstadt, Germany) in PBS at room temperature for $1 \mathrm{~h}$ and subsequently incubated with primary antibodies against GAPDHS (cat. no. ab153802; 1:100 in blocking solution; Abcam, Cambridge, UK), PGK2 (cat. no. ab183031; 1:100 in blocking solution; Abcam, Cambridge, UK) or LDHC (cat. no. ab222910; 1:100 in blocking solution; Abcam, Cambridge, UK) overnight at $4^{\circ} \mathrm{C}$. Subsequently, the sections were washed with PBS three times and incubated with horseradish peroxidase (HRP)-conjugated goat anti-rabbit immunoglobulin (Ig)G (cat. no. ZB2301; Beijing Zhongshan Jinqiao Biotechnology Co., Ltd., Beijing, China) at a final dilution of 1:400 for $1 \mathrm{~h}$ at $37^{\circ} \mathrm{C}$. A DAB kit (OriGene Technologies, Inc., Beijing, China) was used to visualize the positive staining results. Hematoxylin was used to counterstain the sections at room temperature for $1 \mathrm{~min}$, then the sections were dehydrated and mounted onto a Leica DM LB2 light microscope (Leica Microsystems $\mathrm{GmbH}$, Wetzlar, Germany) for bright-field microscopy. The negative controls were processed using pre-immune IgG (1 $\mu \mathrm{g} / \mathrm{ml}$; cat. no. I5006; Sigma-Aldrich; Merck KGaA) as the primary antibody. The sections were examined by bright field microscopy and the mean density of positive immunostaining was analyzed with Image-Pro Plus 6.0 image analysis software (Media Cybernetics, Inc., Rockville, MD, USA).

Immunofluorescence quantitative evaluation of GAPDHS, PGK2 and LDHC in spermatozoa. Cellular localization and quantitative analysis of GAPDHS, PGK2 and LDHC in the spermatozoa were performed using immunofluorescence as previously described (9). The washed spermatozoa were smeared on coverslips that were pre-coated with $1 \%(\mathrm{v} / \mathrm{v})$ gelatin. The air-dried slides were fixed with cold methanol at 
room temperature for $10 \mathrm{~min}$, blocked for $1 \mathrm{~h}$ at room temperature with $3 \%(\mathrm{v} / \mathrm{v}) \mathrm{BSA}$ in PBS and incubated at $37^{\circ} \mathrm{C}$ for $1 \mathrm{~h}$ with the aforementioned primary antibodies against GAPDHS, PGK2 or LDHC, which were diluted 1:50 in PBS containing $3 \%(\mathrm{w} / \mathrm{v}) \mathrm{BSA}$. After washing with PBS, the corresponding secondary antibody [fluorescein isothiocyanate-labelled anti-rabbit IgG; 1:100 in PBS containing 3\% (w/v) BSA] was applied to the slides and they were incubated at room temperature for $1 \mathrm{~h}$. Pre-immune IgG was used as the negative control. Propidium iodide $(0.01 \mathrm{mg} / \mathrm{ml}$; Invitrogen; Thermo Fisher Scientific, Inc., Waltham, MA, USA) counterstaining was performed to visualize the nuclei. The sections were mounted in $80 \%(\mathrm{v} / \mathrm{v})$ glycerol and examined with a Zeiss LSM-510 META confocal laser scanning microscope (Carl Zeiss AG, Oberkochen, Germany).

Quantitative assessment of the protein expression in the spermatozoa was performed using a Zeiss LSM 510 laser confocal microscope (Carl Zeiss AG). The slides were systematically examined at x400 magnification, according to the WHO manual (2010), until a total of 200 spermatozoa had been assessed. Using Zeiss LSM 510 Meta software version 3.2 (Carl Zeiss AG), the fluorescence intensity value per stained cell was calculated automatically by subtracting the background fluorescence intensity, which was determined by scanning a sperm free area.

Sperm motility analysis. Following liquefication of the semen at $37^{\circ} \mathrm{C}$ for $30 \mathrm{~min}, 3 \mu \mathrm{l}$ semen was placed into $20 \mu \mathrm{m}$ deep chambers (2X-CEL; Hamilton Thorne Research, Beverly, MA, USA). The motility parameters, including progressive motility and other parameters of curvilinear velocity, straight-line velocity, average path velocity and linearity were measured and recorded at $37^{\circ} \mathrm{C}$ using computer-aided sperm analyzer (HTM-IVOS; Version 12.3, Hamilton Thorne Research).

Protein extraction. Sperm samples and testis tissues were collected for protein extraction. Briefly, the spermatozoa suspensions were sonicated in radioimmunoprecipitation assay (RIPA) lysis solution (20 mM Tris, pH 7.5; 150 mM NaCl; $1 \%$ Nonidet P-40; $0.5 \%$ sodium deoxycholate; $1 \mathrm{mM}$ EDTA and $0.1 \%$ SDS) and kept on ice for $2 \mathrm{~h}$ for protein lysis. The testicular tissues were ground in liquid nitrogen, solubilized with RIPA lysis buffer and kept on ice for $2 \mathrm{~h}$ for protein lysis. Following lysis, the mixtures were centrifuged at $12,000 \mathrm{x}$ g for $20 \mathrm{~min}$ at $4^{\circ} \mathrm{C}$. The supernatant was collected and precipitated with 4 volumes of ice-cold acetone for $2 \mathrm{~h}$ and centrifugated at $12,000 \mathrm{x} g$ for $20 \mathrm{~min}$ at $4^{\circ} \mathrm{C}$. The precipitate was collected and dissolved in lysis buffer. The solubilized samples were divided into aliquots and stored at $-80^{\circ} \mathrm{C}$ for western blotting.

Western blot analysis. Western blot analysis was used for comparative analysis of the gene expression of GAPDHS, LDHC and PGK2 in the different samples. The proteins samples $(20 \mu \mathrm{g}$ each) were separated by $12 \%$ SDS-PAGE and transferred to polyvinylidene difluoride membranes. After blocking with $5 \%(\mathrm{w} / \mathrm{v})$ skimmed milk for $1 \mathrm{~h}$ at $37^{\circ} \mathrm{C}$, the membranes were co-incubated with the aforementioned primary antibodies used for immunohistochemical analysis under gentle agitation at $4^{\circ} \mathrm{C}$ overnight. Subsequently, the membranes were washed with $0.5 \%$ (v/v) Tween-20 in TBS
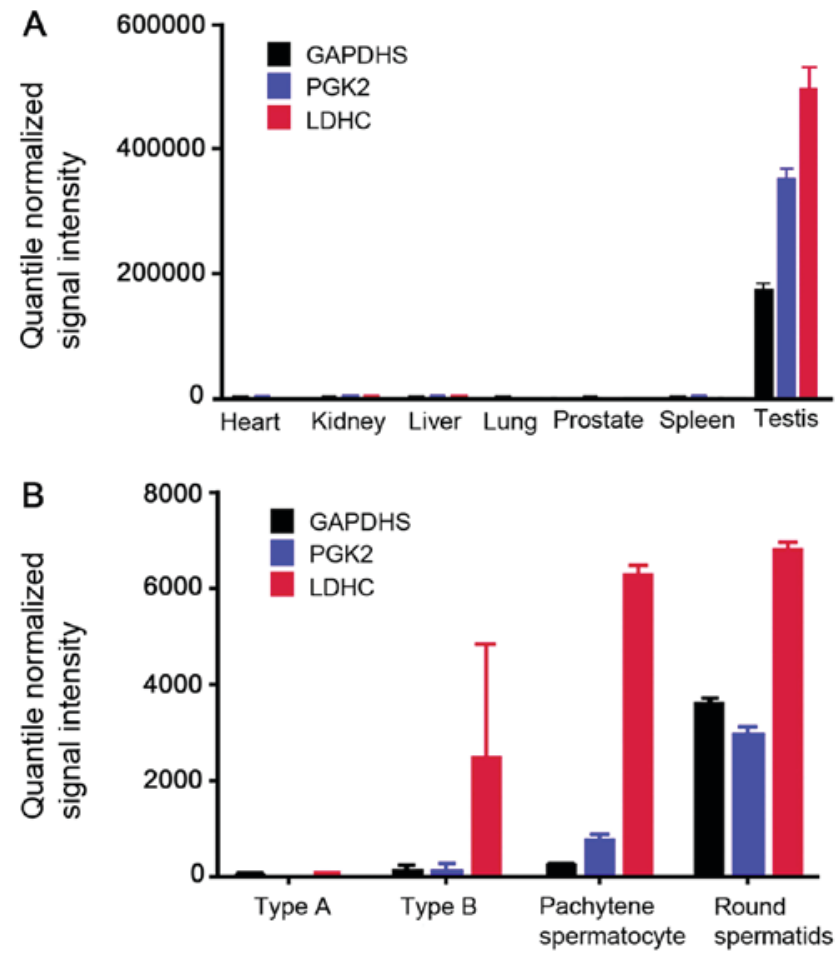

Figure 1. Differential expression of GAPDHS, PGK2 and LDHC in human tissues and testicular germ cells. (A and B) Gene expression values from microarray data were re-analyzed from data sets (A) GDS3113 (human) and (B) GDS2390 (murine) from the Gene Expression Omnibus database by retrieving gene symbols in data analysis tools and plotted. GAPDHS, glyceraldehyde-3-phosphate dehydrogenase, testis-specific; LDHC, lactate dehydrogenase C; PGK2, phosphoglycerate kinase 2.

three times and subsequently incubated with HRP-conjugated goat anti-rabbit or goat anti-mouse IgG (cat. nos. ZB2301 and ZB2305, respectively; Beijing Zhongshan Jinqiao Biotechnology Co., Ltd.) for $1 \mathrm{~h}$ at room temperature. The immune-reactive complexes were detected using an Enhanced Chemiluminescence kit (GE Healthcare, Chicago, IL, USA). GeneTools (version 4.02; Syngene Europe, Cambridge, UK) was used to analyze the density of each reaction band. The results are presented as the relative quantity of target proteins compared with $\beta$-actin (cat. no. sc-81178; 1:200 in blocking solution; Santa Cruz Biotechnology, Inc., Dallas, TX, USA) or $\beta$-tubulin (cat. no. sc-5274; 1:200 in blocking solution; Santa Cruz Biotechnology, Inc.).

Statistical analysis. Data are presented as the mean \pm standard deviation. The means of two groups were analyzed using a Student's t-test and the means of more than two groups were analyzed by one-way analysis of variance and Newman-Keuls was used to compare all groups. Pearson's correlation was performed to identify correlation coefficient. GraphPad Prism 7 (GraphPad Software, Inc., La Jolla, CA, USA) was used to perform the statistical analysis. $\mathrm{P}<0.05$ was considered to indicate a statistically significant difference.

\section{Results}

In silico bioinformatics analysis of GAPDHS, PGK2 and LDHC in the testes. Gene expression profiles of the GDS3113 

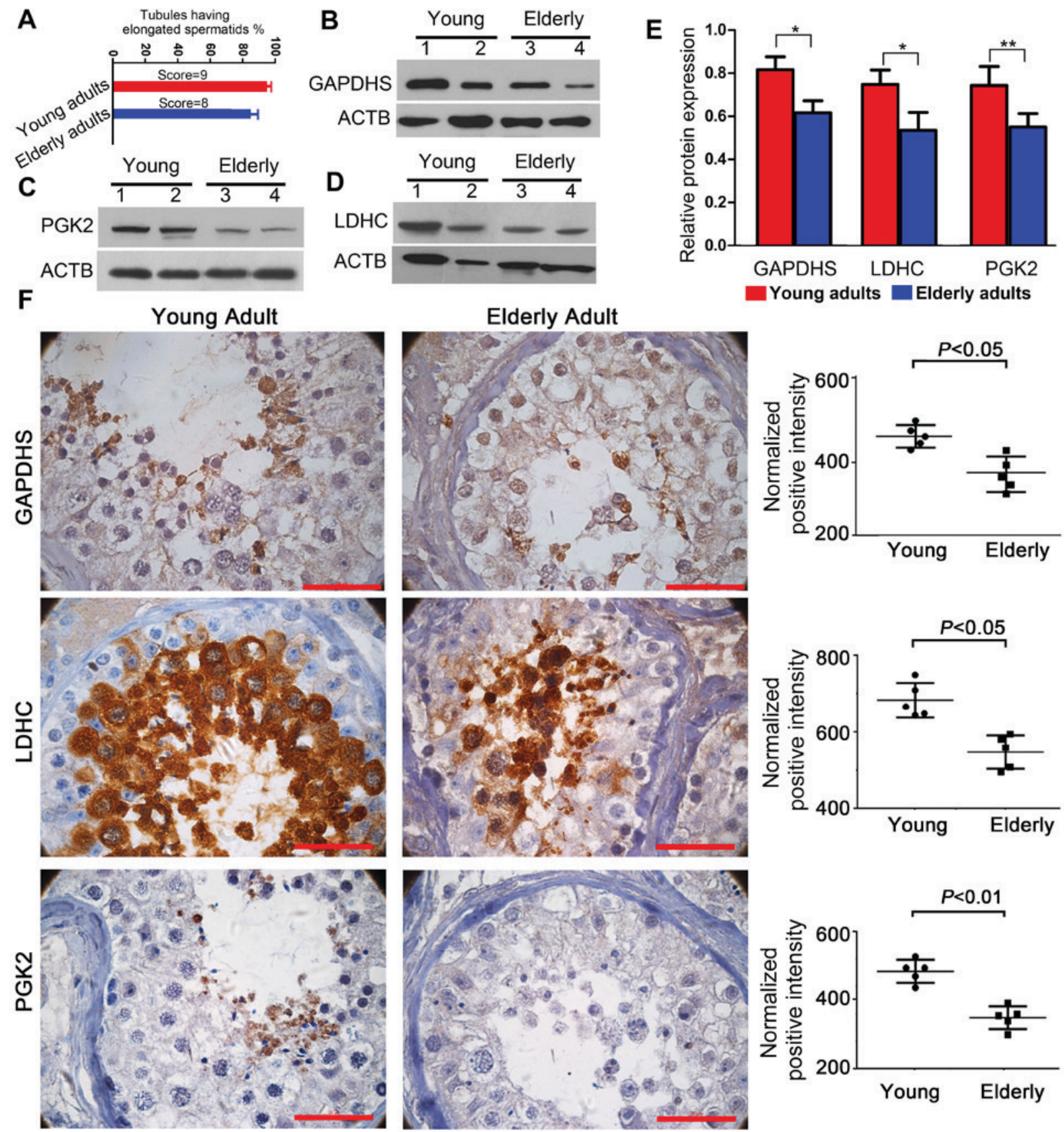

Figure 2. Expression and cellular localization of GAPDHS, PGK2 and LDHC in testes from young adults and elderly adults. (A-F) The testes of five samples from young adults and five from elderly men were (A) evaluated by Johnsen scoring method and analyzed by (B-E) western blotting and (F) immunohistochemistry; scale bar, $20 \mu \mathrm{m}$. The expression between young adult and elderly adult were compared statistically by Student's t-test; ${ }^{*} \mathrm{P}<0.05$ and ${ }^{* * *} \mathrm{P}<0.01$. ACTB, $\beta$-actin; GAPDHS, glyceraldehyde-3-phosphate dehydrogenase, testis-specific; LDHC, lactate dehydrogenase C; PGK2, phosphoglycerate kinase 2.

dataset were extracted from the GEO Database, which included the gene expression values of different human tissues. The expression values of GAPDHS, PGK2 and LDHC in the main tissues (heart, kidney, liver, lung, prostate, spleen and testis) were downloaded and re-compared. The results demonstrated that GAPDHS, PGK2 and LDHC were specifically expressed in the testis (Fig. 1A). The GDS2390 dataset included murine gene expression information from different germ cells of type A and type B spermatogonia, spermatocytes and round spermatids (Fig. 1B). The comparative analysis demonstrated that GAPDHS and PGK2 were mainly expressed in the round spermatids, whereas LDHC was mainly expressed in the spermatocytes and round spermatids. The results indicated that GAPDHS, PGK2 and LDHC are post-meiotic germ cell-specific genes in the testes.

Cellular localization of GAPDHS, PGK2 and LDHC in the human testes. The score evaluation of the testicular sections demonstrated that the testes of young adults contained normal spermatogenesis (score $=9$ ), whereas the testes from elderly adults exhibited atrophic morphology $($ score $=8)$ (Fig. 2A). Western blot analysis demonstrated that the protein expression levels of GAPDHS, PGK2 and LDHC were lower in elderly testes compared with young adults (Fig. 2B-E). 
Table I. Association between expression of GAPDHS, PGK2 and LDHC in ejaculated sperm and progressive sperm motility and sperm concentration.

\begin{tabular}{lccccc}
\hline & \multicolumn{2}{c}{$\begin{array}{c}\text { Progressive } \\
\text { sperm motility }\end{array}$} & & \multicolumn{2}{c}{$\begin{array}{c}\text { Sperm } \\
\text { concentration }\end{array}$} \\
\cline { 2 - 3 } \cline { 5 - 6 } Parameter & $\mathrm{R}$ & P-value & & $\mathrm{R}$ & P-value \\
\hline GAPDHS & 0.53 & $<0.05$ & & 0.49 & $<0.05$ \\
PGK2 & 0.62 & $<0.05$ & & 0.54 & $<0.05$ \\
LDHC & 0.51 & $<0.05$ & & 0.39 & $\mathrm{NS}$ \\
\hline
\end{tabular}

GAPDHS, glyceraldehyde-3-phosphate dehydrogenase, testis-specific; LDHC, lactate dehydrogenase C; PGK2, phosphoglycerate kinase 2; NS, not significant.

Immunohistochemical analysis results demonstrated that GAPDHS, PGK2 and LDHC were expressed in specific localizations in the testicular germ cells. GAPDHS and PGK2 were mainly located in the round spermatids, whereas LDHC was mainly observed in the spermatocytes and round spermatids (Fig. 2F). The images in Fig. 2F also indicated that, compared with the tests from young adults, there were notable morphological alterations in testes of the elderly adults, including an irregular arrangement of the germ cells and an increase in the mesenchymal cell layers; these morphological changes agreed well with the score evaluation of the testicular sections. These cellular localizations were also consistent with those of the bioinformatics analysis. The results demonstrated an age-associated reduction in the expression levels of GAPDHS, PGK2 and LDHC in the human testes, which suggested that the expression levels are closely associated with spermatogenesis.

Quantitative expression of GAPDHS, PGK2 and LDHC in sperm. The quantified expression levels of GAPDHS, PGK2 and LDHC in the sperm were closely associated with sperm quality. The expression of GAPDHS and PGK2 in the sperm was significantly associated with the concentration of sperm (Table I). PGK2 had higher correlation coefficients with progressive sperm motility (0.62) and sperm concentration $(0.54)(\mathrm{P}<0.05$; Table I).

Immature spermatozoa were further isolated from the spermatozoon populations. Significantly different proportions of immature spermatozoa were present in the normozoospermia $(23.15 \pm 2.54 \%)$ and asthenozoospermia $(59.14 \pm 3.09 \%)$ group $(\mathrm{P}<0.05$; Fig. 3A). The immature spermatozoa and asthenozoospermic spermatozoa exhibited levels of significantly lower progressive motility than the normozoospermic spermatozoa (immature, 24.2 $\pm 1.68 \%$; asthenozoospermia, 18.9 $\pm 2.08 \%$; normozoospermia, $62.3 \pm 3.19 \%$; P<0.05; Fig. 3B). Immunofluorescence quantitative analysis of GAPDHS, PGK2 and LDHC in the spermatozoa indicated that GAPDHS, PGK2 and LDHC were mainly localized in the principal piece of the sperm, in which the energy for sperm motility is produced through glycolysis (Fig. 3C; representative PGK2 expression, which is similar to GAPDHS and LDHC expression patterns). Significantly lower percentages of positive expression and
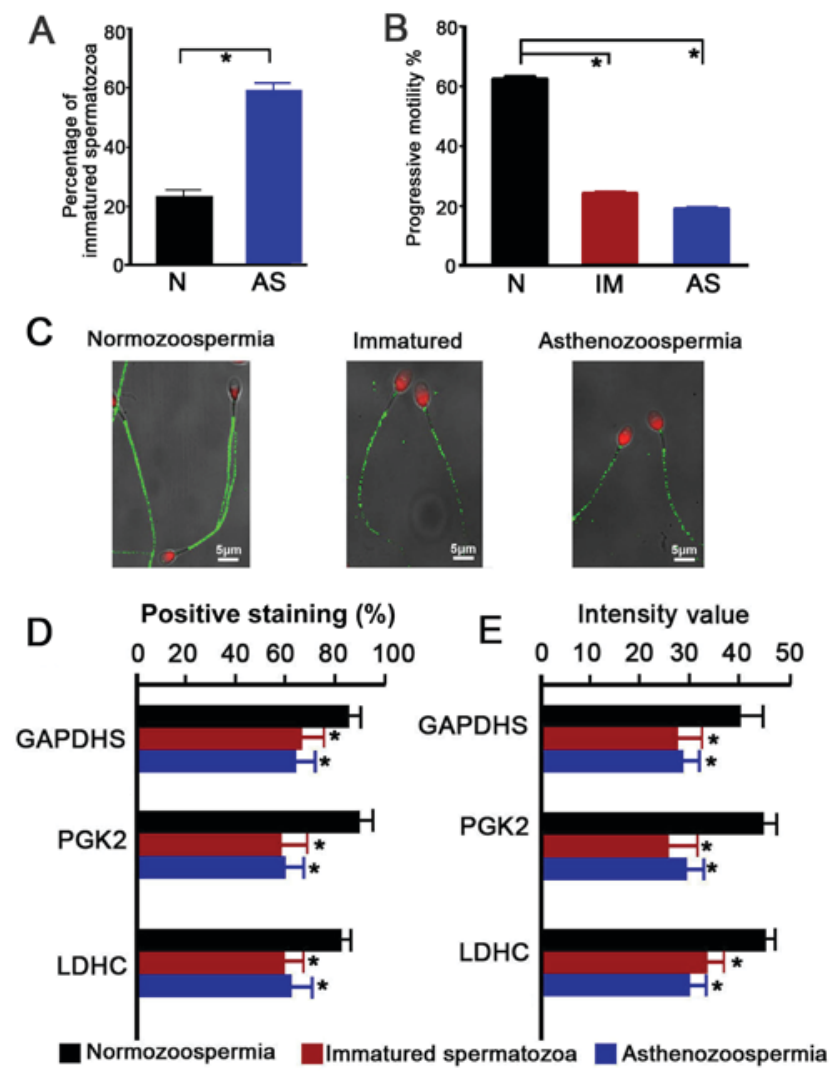

Figure 3. Immunofluorescence localization and intensity of GAPDHS, PGK2 and LDHC in ejaculated spermatozoa of normal, immature and asthenozoospermia samples. (A) Percentage of immature spermatozoa in normal and asthenozoospermia samples. (B) Percentage of progressive motility of spermatozoa from normal, immature and asthenozoospermia samples. (C) Representative images of PGK2 (green) expression localization in spermatozoa; scale bar, $5 \mu \mathrm{m}$; nuclei were stained with propidium iodide (red) (D and E) Quantitative analysis of (D) percentage of positive-stained cells and (E) fluorescence intensity of GAPDHS, PGK2 and LDHC on spermatozoa. Data were compared by one-way analysis of variance; ${ }^{*} \mathrm{P}<0.05$ vs. N. AS, asthenozoospermia; GAPDHS, glyceraldehyde-3-phosphate dehydrogenase, testis-specific; IM, immature spermatozoa; LDHC, lactate dehydrogenase C; $\mathrm{N}$, normozoospermia; PGK2, phosphoglycerate kinase 2.

fluorescence intensities were observed in the immature spermatozoa and asthenozoospermic spermatozoa $(\mathrm{P}<0.05$; Fig. 3D and E, respectively).

Western blot analysis revealed similarly reduced expression levels of GAPDHS, PGK2 and LDHC in the asthenozoospermic spermatozoa and immature sperm from the normal young adults (Fig. 4).

\section{Discussion}

Spermatozoa are produced in the testes and mature in the epididymis. The sperm population of each ejaculation is heterogeneous and includes mature and immature spermatozoa (19). In the present study it was hypothesized that different proportions of mature and immature spermatozoa contribute to the phenotype of poor sperm quality in patients with asthenozoospermia and elderly males, and therefore there is a similar mechanism underlying the poor sperm quality of immature and asthenozoospermic spermatozoa.

Multiple signaling and metabolic molecules are highly expressed in the testes and are likely to contribute to male 


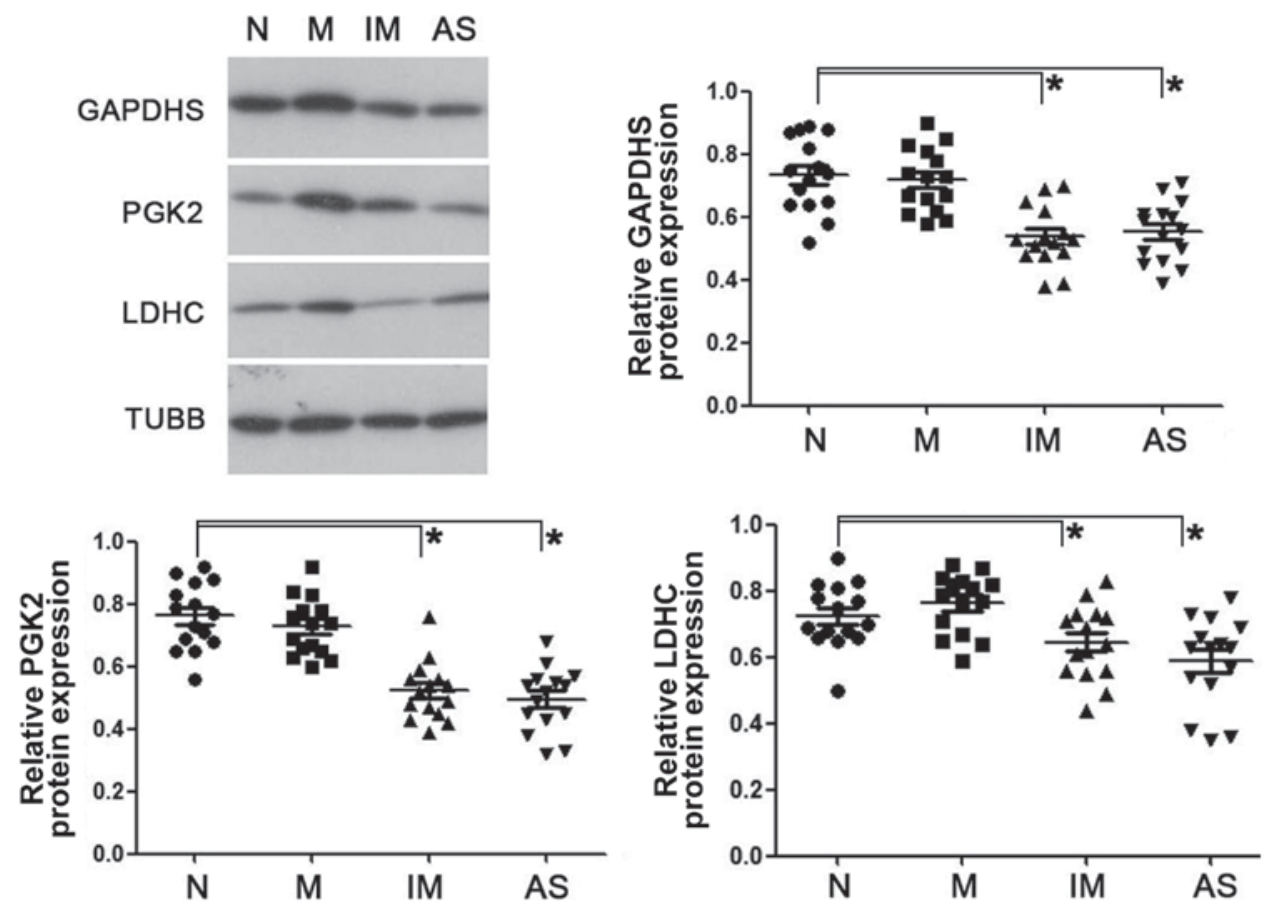

Figure 4. Western blot analysis of GAPDHS, PGK2 and LDHC on ejaculated spermatozoa in normal, mature, immature and asthenozoospermia samples. Data were compared by one-way analysis of variance; "P<0.05. AS, asthenozoospermia; GAPDHS, glyceraldehyde-3-phosphate dehydrogenase, testis-specific; IM, immature spermatozoa; M, matured spermatozoa; N, normozoospermia; PGK2, phosphoglycerate kinase 2; LDHC, lactate dehydrogenase C; TUBB, $\beta$-tubulin.

infertility (20). Glycolysis is the main energy source for sperm motility. GAPDHS, PGK2 and LDHC are the key enzymes involved in glycolysis. Disruption of these enzymes leads to aberrant ATP production and sperm motility. However, their association with sperm quality requires investigation prior to their clinical application as promising diagnostic or contraceptive targets $(21,22)$. In the present study, the key glycolytic enzymes GAPDHS, PGK2 and LDHC were comprehensively characterized in the human testis and spermatozoa, which provided novel information for understanding the regulation of sperm quality.

As revealed by in silico analysis, GAPDHS, PGK2 and LDHC are specifically expressed in the testes. GAPDHS and PGK2 were mainly expressed in the post-meiotic germ cells, particularly in the round spermatids, and LDHC was mainly expressed in the spermatocytes and round spermatids. The expression pattern was further confirmed by immunohistochemical analysis of the testes from young and elderly adults. In addition, GAPDHS, PGK2 and LDHC were mainly localized in the principal piece of the sperm, in which the energy is produced for sperm motility by glycolysis. The tail localization of these proteins in sperm and the importance of the glycolytic pathway in supplying energy for human sperm motility suggests that these proteins serve an important role in sperm motility (23).The comprehensive characteristics of GAPDHS, PGK2 and LDHC in human testes and sperm provides information for clinical applications.

Compared with the testes from the young adults, GAPDHS, PGK2 and LDHC exhibited reduced expression intensities in the testes from the elderly adults in the present study. Most prostate cancer patients have normal spermatogenesis (24), in the present study testes were selected with
Johnsen score $>6$. There may be excess oxidative stress and a reduction in androgen in the testes of elderly males that result in clear morphological differences, including reduced spermatogenesis and loose organization of germ cells (25). The authors' previous study indicated that aged testes are a good model for the identification of age-associated spermatogenesis-associated proteins (26). The negative effects of aging on testes may contribute to altered expression levels of proteins associated with key biological processes. The present results indicated that GAPDHS, PGK2 and LDHC are associated with spermatogenesis. As described in the authors' previous study (9), there was no statistical difference of spermatozoa morphology among the young adults, elderly adults and asthenozoospermia patients; however, similar lower sperm quality (lower progressive sperm motility) was present in spermatozoa of elderly adults and asthenozoospermia patients. Our previous study also revealed that there were lower expression levels of PGK2 in sperm from males or asthenozoospermic patients compared with sperm from healthy young males. Sperm functional analysis in a previous study validated the close association between the expression of PGK2 and sperm motility (9). In the present study, normal semen samples were divided into mature and immature sperm. The mature sperm exhibited good motility and morphology, whereas the immature population exhibited poor sperm quality that was similar to the spermatozoa of the asthenozoospermic patients. The sperm intensity value and localization percentage of GAPDHS, PGK2 and LDHC were downregulated in the immature and asthenozoospermic spermatozoa. These results suggested that expression levels of GAPDHS, PGK2 and LDHC in sperm were associated with sperm quality, and immature and asthenozoospermic 
spermatozoa may share similar molecular mechanisms. The results indicated that asthenozoospermia may involve an increased percentage of immature sperm, which may lead to poor sperm quality. The common mechanism underlying poor sperm quality requires further investigation.

The authors' previous study suggested that a similar mechanism underlying poor sperm quality may exist in elderly adults and patients with asthenozoospermia (9). There may be gene expression variations occurring in elderly adults and asthenozoospermic patients. In the present study, it was demonstrated that there is reduced expression of GAPDHS, PGK2 and LDHC in asthenozoospermic and immature spermatozoa compared with normozoospermic males. The present study hypothesized that the expression of testis-specific genes undergo complex and sophisticated regulation in different spermatogenic cells at specific stages. Suboptimum expression of certain genes may contribute to poor-quality spermatozoa suffering from an abnormal process of sperm formation.

The results of the present study indicated that GAPDHS, PGK2 and LDHC were associated with sperm quality and function. These enzymes catalyze successive steps in glycolysis and loss of any will eliminate ATP production via this pathway. Systematic analysis of GAPDHS, PGK2 and LDHC may improve the understanding of the metabolism-dependent signaling events required for fertilization. The present study indicated that a similar molecular mechanism of poor sperm quality maybe shared by immature and asthenozoospermic spermatozoa. These results may provide novel insights into the mechanisms underlying sperm quality and may contribute to the diagnosis and treatment of asthenozoospermia. Prior to any clinical application of these molecules as biomarkers for the diagnosis of male infertility, additional cases should be used to assess relevance with sperm quality and evaluate reliability of quantitative methods.

\section{Acknowledgements}

Not applicable.

\section{Funding}

The present study was supported by The National Natural Science Foundation of China (grant no. 81741027), The Clinical Research Special Fund of Chinese Medical Association (grant no. 17020160685) and The Key Research and Development Plan of Yantai (grant no. 2017YT06000491).

\section{Availability of data and materials}

The datasets used and/or analyzed during the current study are available from the corresponding author on reasonable request.

\section{Authors' contributions}

FJL conceptualized the study design and drafted the manuscript. XXL, QL and WJW acquired and analyzed the data, and revised the manuscript. All authors reviewed and approved the final manuscript.

\section{Ethics approval and consent to participate}

The present study was approved by the Ethics Committee of Qingdao University Affiliated Yuhuangding Hospital (Yantai, China). All participants provided informed consent.

\section{Patient consent for publication}

Not applicable.

\section{Competing interests}

The authors declare that they have no competing interests.

\section{References}

1. Li J, Liu F, Liu X, Liu J, Zhu P, Wan F, Jin S, Wang W, Li N, Liu J and Wang H: Mapping of the human testicular proteome and its relationship with that of the epididymis and spermatozoa. Mol Cell Proteomics 10: M110.004630, 2011.

2. Feng HL: Molecular biology of male infertility. Arch Androl 49: 19-27, 2003.

3. Curi SM, Ariagno JI, Chenlo PH, Mendeluk GR, Pugliese MN, Sardi Segovia LM, Repetto HE and Blanco AM: Asthenozoospermia: Analysis of a large population. Arch Androl 49: 343-349, 2003.

4. Ortega C, Verheyen G, Raick D, Camus M, Devroey P and Tournaye H: Absolute asthenozoospermia and ICSI: What are the options? Hum Reprod Update 17: 684-692, 2011.

5. Sullivan R and Mieusset R: The human epididymis: Its function in sperm maturation. Hum Reprod Update 22: 574-587, 2016.

6. Narisawa S, Hecht NB, Goldberg E, Boatright KM, Reed JC and Millán JL: Testis-specific cytochrome c-null mice produce functional sperm but undergo early testicular atrophy. Mol Cell Biol 22: 5554-5562, 2002.

7. Margaryan H, Dorosh A, Capkova J, Manaskova-Postlerova P, Philimonenko A, Hozak P and Peknicova J: Characterization and possible function of glyceraldehyde-3-phosphate dehydrogenase-spermatogenic protein GAPDHS in mammalian sperm. Reprod Biol Endocrinol 13: 15, 2015.

8. Odet F, Gabel SA, Williams J, London RE, Goldberg E and Eddy EM: Lactate dehydrogenase $\mathrm{C}$ and energy metabolism in mouse sperm. Biol Reprod 85: 556-564, 2001.

9. Liu XX, Zhang H, Shen XF, Liu FJ, Liu J and Wang WJ: Characteristics of testis-specific phosphoglycerate kinase 2 and its association with human sperm quality. Hum Reprod 31: 273-279, 2016.

10. Goldberg E: Reproductive implications of LDH-C4 and other testis-specific isozymes. Exp Clin Immunogenet 2: 120-124, 1985.

11. Coonrod S, Vitale A, Duan C, Bristol-Gould S, Herr J and Goldberg E: Testis-specific lactate dehydrogenase (LDH-C4; Ldh3) in murine oocytes and preimplantation embryos. J Androl 27: 502-509, 2006.

12. Wei D, Wei L, Li X, Wang Y and Wei L: Effect of hypoxia on Ldh-c expression in somatic cells of Plateau Pika. Int J Environ Res Public Health 13: pii: E773, 2016.

13. Miki K, Qu W, Goulding EH, Willis WD, Bunch DO, Strader LF, Perreault SD, Eddy EM and O'Brien DA: Glyceraldehyde 3-phosphate dehydrogenase-S, a sperm-specific glycolytic enzyme, is required for sperm motility and male fertility. Proc Natl Acad Sci USA 101: 16501-16506, 2004.

14. Odet F, Duan C, Willis WD, Goulding EH, Kung A, Eddy EM and Goldberg E: Expression of the gene for mouse lactate dehydrogenase $\mathrm{C}$ (Ldhc) is required for male fertility. Biol Reprod 79: 26-34, 2008.

15. Danshina PV, Geyer CB, Dai Q, Goulding EH, Willis WD, Kitto GB, McCarrey JR, Eddy EM and O'Brien DA: Phosphoglycerate kinase 2 (PGK2) is essential for sperm function and male fertility in mice. Biol Reprod 82: 136-145, 2010.

16. Bergmann M and Kliesch S: Hodenbiopies In: Krause W, Weidner W (eds) Andrologie. Enke Verlag, Stuttgart: pp66-71, 1998.

17. Kovanci E, Kovacs T, Moretti E, Vique L, Bray-Ward P, Ward DC and Huszar G: FISH assessment of aneuploidy frequencies in mature and immature human spermatozoa classified by the absence or presence of cytoplasmic retention. Hum Reprod 16: 1209-1217, 2001 
18. Liu XX, Shen XF and Liu FJ: Screening targeted testis-specific genes for molecular assessment of aberrant sperm quality. Mol Med Rep 14: 1594-1600, 2016.

19. Moustafa MH, Sharma RK, Thornton J, Mascha E, Abdel-Hafez MA, Thomas AJ Jr and Agarwal A: Relationship between ROS production, apoptosis and DNA denaturation in spermatozoa from patients examined for infertility. Hum Reprod 19: 129-138, 2004.

20. Huang Z, Danshina PV, Mohr K, Qu W, Goodson SG, O'Connell TM and O'Brien DA: Sperm function, protein phosphorylation, and metabolism differ in mice lacking successive sperm-specific glycolytic enzymes. Biol Reprod 97: 586-597, 2017.

21. Danshina PV, Qu W, Temple BR, Rojas RJ, Miley MJ, Machius M, Betts L and O'Brien DA: Structural analyses to identify selective inhibitors of glyceraldehyde 3-phosphate dehydrogenase-S, a sperm-specific glycolytic enzyme. Mol Hum Reprod 22: 410-426, 2016

22. Odet F, Gabel S, London RE, Goldberg E and Eddy EM: Glycolysis and mitochondrial respiration in mouse LDHC-null sperm. Biol Reprod 88: 95, 2013.
23. Nascimento JM, Shi LZ, Tam J, Chandsawangbhuwana C, Durrant B, Botvinick EL and Berns MW: Comparison of glycolysis and oxidative phosphorylation as energy sources for mammalian sperm motility, using the combination of fluorescence imaging, laser tweezers, and real-time automated tracking and trapping. J Cell Physiol 217: 745-751, 2008.

24. Moody JA, Ahmed K, Horsfield C, Pedersen MRV, Yap T and Shabbir M: Fertility preservation in testicular cancer-predictors of spermatogenesis. BJU Int 122: 236-242, 2008.

25. Jankovic Velickovic L and Stefanovic V: Hypoxia and spermatogenesis. Int Urol Nephrol 46: 887-894, 2014.

26. Liu FJ, Liu X, Han JL, Wang YW, Jin SH, Liu XX, Liu J, Wang WT and Wang WJ: Aged men share the sperm protein PATE1 defect with young asthenozoospermia patients. Hum Reprod 30:861-869, 2015 .

This work is licensed under a Creative Commons Attribution-NonCommercial-NoDerivatives 4.0 International (CC BY-NC-ND 4.0) License. 